\title{
Optimum Route Selection for Vehicle Navigation
}

\author{
Dalip \\ Department of Information Technology \\ MMEC, Maharishi Markandeshwar University \\ Ambala, India
}

\author{
Vijay Kumar \\ Department of Computer Science and Engineering \\ MMEC, Maharishi Markandeshwar University \\ Ambala, India
}

\begin{abstract}
The objective of Optimum Route Selection for Vehicle Navigation System (ORSVNS) article is to develop a system, which provides information about real time alternate routes to the drivers and also helps in selecting the optimal route among all the alternate routes from an origin to destination. Two types of query systems, special and general, are designed for drivers. Here, the criterion for route selection is introduced using primary and secondary road attributes. The presented methodology helps the drivers in better decision making to choose optimal route using fuzzy logic. For experimental results ORSVNS is tested over $220 \mathbf{~ k m}$ portion of Haryana state in India.

Keywords-Vehicle Navigation; Route Selection; Fuzzy; Optimum; Navigation System
\end{abstract}

\section{INTRODUCTION}

Today, enhancement in advance technologies (communication, sensors, microelectronics and information technology) is technically possible to provide the real time [1] [16] information of traffic conditions to drivers. A Dynamic Route Guidance (DRG) system provides the actual route advice to drivers in real time traffic condition to reduce the traffic delays. This system is implemented in Indian road context, which are categorized in the following parts- Express Way, National Highways, State Highways, Urban Roads and Rural Roads. National Highways and Express ways are the most preferred by the most of the DRG system routes to reach the destination. As there is no Express Way in Haryana (experimental study), it is not included in the presented work. The traffic volume is increasing every year. The production of automobiles in India is in millions of [2] units as shown in Fig. 1 , which is the main cause of traffic congestion. The other causes are poor road condition, poor traffic management and heterogeneous mode of transport (Heavy goods vehicle, Trunk, Bus, Mini Bus, Cab and motorcycle etc.). There are three modes of route selection- short, economical and easy to travel. The route with the shortest distance is recommended in short mode. In case of economy mode, the most economical path in terms of fuel, toll plaza and vehicle maintenance cost is recommended. In easy mode, a route is selected which is suitable for easy driving. Firstly, short, mode is most commonly used mode in all DRG systems.

An alternate route provides [3] additional capacity to service primary route traffic. Alternate routes start from one point on primary route and ends at another point as shown in Fig. 2. Some events like the traffic clogging, emergency, natural disasters, traffic incidents, snowstorm, fog, flood, road blockage, forgotten route by drivers are responsible for alternate routes.

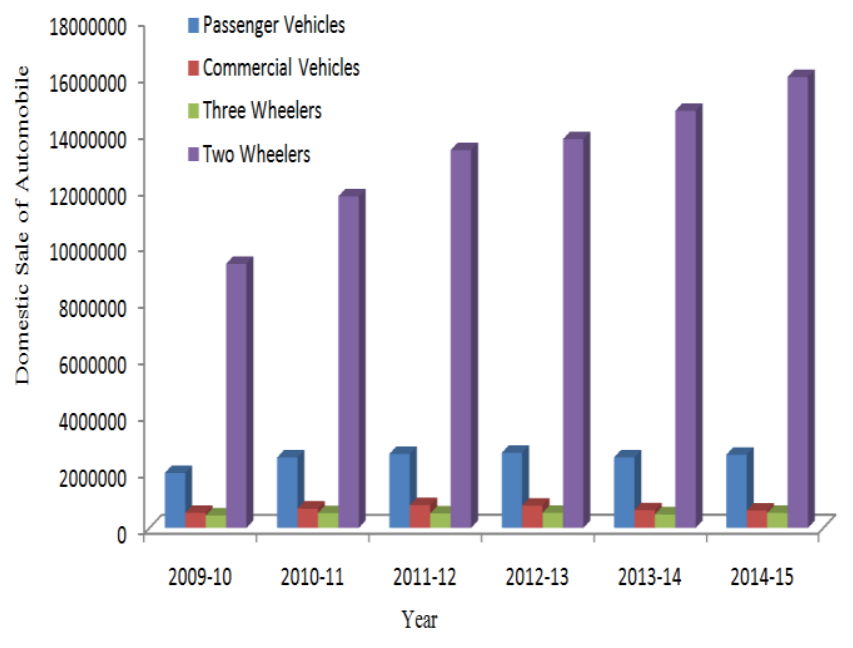

Fig. 1. Domestic Sales of Automobile in India [Source: SLAM]

Fig. 2 shows a road network, which represents three alternate routes with beginning and terminal point on the primary route. In case of blockage of selected route there is need of route diversion and reassignment, which is represented in Fig. 3. Planned events like major roadway construction and maintenance, and unplanned events like traffic incidents, emergency roadwork, adverse weather and emergency are the causes for alternate route plan. The congestion impacts of these events on road capacity are blocked travel lane(s) or road segment, reduce traffic speed and capacity.

A large number of route guidance, alternate route selection, optimum route selection and route diversion systems (Peter Bonsall et al. (1992), Kim et al. (2003), Mohamed Abdel-Aty et al. (2004), Shou-Ren HU et al. (2005), Hey Ran Kim et al. (2005), Alexander Aved et al. (2007), S.S. Keshkamat (2008), Constantinos Antonioua et al. (2011), Anastasia Spiliopoulou et al. (2013), Xingang Li et al. (2014)) have been developed to select the optimum route but a limited technical literature for selecting optimal route to the best of our knowledge is presented here. Peter Bonsall designed a route guidance [4] and information system on route choice for urban networks. Advanced Traveler Information Systems (ATIS) provided [5] alternate routes to driver using generalized estimating equations and binomial probit link function. Time taken to travel on diverted route is less as compared to on normal route thus it increases the probability of diversion. Real-Time Route Diversion Management System (RTRDS) was implemented to [6] create optimal route diversions using available real-time and historical traffic information. 


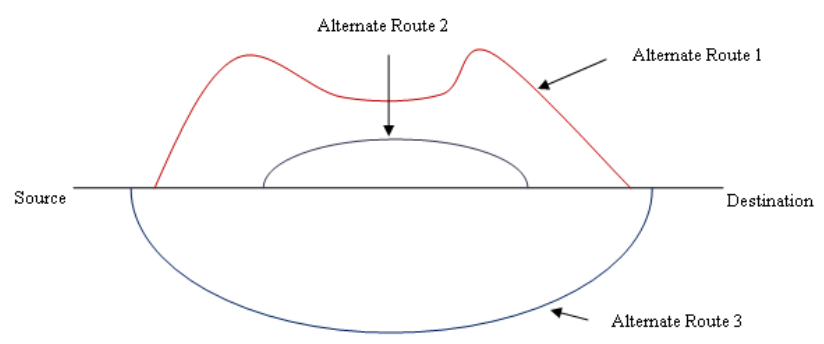

Fig. 2. Road Network

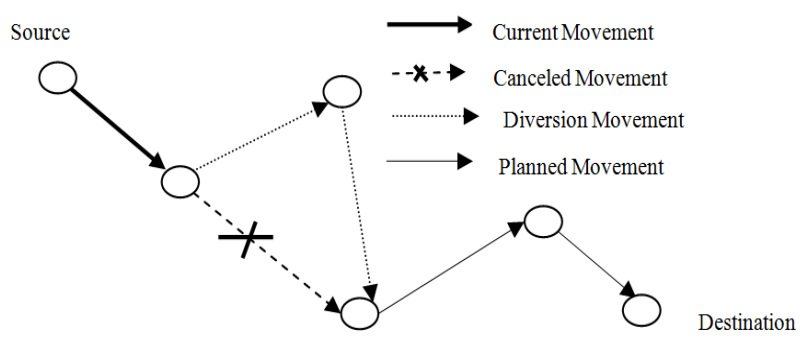

Fig. 3. Route Diversion and Reassignment

A flexible and cost-effective systematic framework based [7] on traffic assignment models was designed to analyze the value of traffic information in route diversion control scheme. With the use of online traffic management system, other dynamic route diversion model [8] is developed to capture travelers' route and their switching behavior in real time Adaptive Kalman Filtering technique is also used to present freeway O-D pattern prediction algorithm. The framework presented by Constantinos Antonioua et al. has discussed the impact of predictive guidance in reducing average travel time and travel time variability during incidents. The predictive Variable Message Signs (VMS) [9] shifts a significant number of vehicles from routes taking higher travel time to routes taking lower travel time, showing more consequently reduction in delays and efficient utilization of the network capacity. The contribution of onsite information [10] is to estimate the influence of information on drivers' route diversion decision and network performance accurately. To avoid the [11] recurrent motorway congestion, two route guidance policies have been developed, which is triggered by a saturated offramp, but is not efficient to resolve the spread out and motorway congestion problem. To resolve this, a second case based [12] on user-optimum considerations was implemented. Xingang Li et al. designed the diversion drivers' [13] from expressway to analyze the behavior under different conditions of network and traffic, to find the key factors influencing diversion behavior. Multi criteria methods for high [14] speed rail route selection presented by Mohd Rapik Saat et al. for Malaysia. These methods provide a set of tools to analyze and compare the different alternatives for a rail transport route. It is found from the above review of literature that there is a need of existing as well as implementation of new systems, which will help the system users to select more precise optimal route among all alternate routes on the basis of vehicle type. A fuzzy logic based decision making system is designed to achieve our goal which displays optimal route using experienced based fuzzy score of primary and secondary road attributes.

The rest of the paper is organized as follows. The section I describes introduction and the related work for optimal route selection for vehicle navigation system. The section II presents case study from origin (MM University, Mullana) to destination (Karnal) of Haryana state, India. The road characteristic and discussion on primary and secondary road characteristics is provided in section III. The optimal route selection criterion is discussed in section IV. Section V presents the results and discussion of implemented work. Finally section 6 shows the conclusion and future work of this paper.

\section{CASE STUdY: ANALYSIS OF ROUTES FROM MM UNIVERSITY TO KARNAL}

From an origin (MM University, Mullana) to destination (Karnal) route is taken with three alternate routes to present the specific problem in this case study. Table I presents route description of one original and three possible alternate routes of Haryana, India. The distance pair between the cities is shown in Table II which is used in analysis of routes from origin to destination. As described in Table II, the distances between two same cities remain zero. Entries below zero shows distance between city pairs in kilometers, miles and nautical miles but entries above zero are blank due to repetition of city pair. Traffic congestion may be more during starting and ending session of the companies and educational institutes. Thus the volume of automobile traffic is higher than the usual at that duration of time. As the driver already knows all feasible routes for a trip, there is a need of selecting optimal route and during the time of journey, before each road junction, the driver has the options to evaluate alternate routes. The driver has to take decision to choose alternate route on the basis of road attribute values. The evaluation of route is measured by number of factors. For instance, if the driver wants to reach his/her destination as soon as possible, the dominant factor would be travel time of all feasible routes from origin to destination.

In case of Route1 blockage or traffic congestion on Route1, alternate routes will be considered, but the problem in choosing more precise optimal route among all alternate routes according to vehicles type arises here. For example Route4 will not be well suitable for heavy vehicles in term of several road attributes like "bridge clearance", "bridge load", "unnecessary detours" and "congested road". The following research gaps are analyzed in this case study.

- No criteria for selecting route according to transportation mode

- No query system designed for general and special query

- No provision for selecting more precise optimal route

The solution of these research gaps and the criteria for optimum route selection are explained in the further sections. 
TABLE I. ROUTE DESCRIPTION OF MM UNIVERSITY TO KARNAL

\begin{tabular}{lllll}
\hline & $\begin{array}{l}\text { Original/ } \\
\text { Alternate }\end{array}$ & Stop List & Via & Route Type \\
\hline Route1 & Original & [MM University, Barara, Sahabad, Pipli, Nilokheri, Trarori, Karnal] & Barara & $\begin{array}{l}\text { [Urban+ National } \\
\text { Highway] }\end{array}$ \\
& & [MM University, Mullana, Kalpi, Saha, Sahabad, Pipli, Nilokheri, & Saha & [Urban+ National \\
Route2 & Alternate & $\begin{array}{l}\text { Highway] } \\
\text { Trarori, Karnal] }\end{array}$ & Yamuna & [ Urban Only] \\
Route3 & Alternate & Radaur, Ladwa, Indri, Rambha Karnal] & Nagar & Babain \\
Route4 & Alternate & [MM University, Barara, Babain, Ladwa, Indri, Rambha Karnal] & Rural] \\
\hline
\end{tabular}

TABLE II. Distance Between City Pairs of Haryana State, India

\begin{tabular}{|c|c|c|c|c|c|c|c|c|}
\hline & $\begin{array}{l}\text { Yamuna } \\
\text { Nagar }\end{array}$ & Amabala Cantt & Kurukshetra & Karnal & Panipat & Hisar & Rohatak & Jind \\
\hline \multicolumn{9}{|l|}{ (a) In Kilometers } \\
\hline Yamuna Nagar & 0 & & & & & & & \\
\hline Ambala Cantt & 58 & 0 & & & & & & \\
\hline Kurukshetra & 45 & 46 & 0 & & & & & \\
\hline Karnal & 66 & 81 & 37 & 0 & & & & \\
\hline Panipat & 99 & 120 & 71 & 36 & 0 & & & \\
\hline Hisar & 206 & 190 & 162 & 156 & 142 & 0 & & \\
\hline Rohatak & 180 & 226 & 177 & 116 & 77 & 90 & 0 & \\
\hline Jind & 149 & 151 & 103 & 84 & 68 & 76 & 59 & 0 \\
\hline \multicolumn{9}{|l|}{ (b) In Miles } \\
\hline Yamuna Nagar & 0 & & & & & & & \\
\hline Ambala Cantt & 58 & 0 & & & & & & \\
\hline Kurukshetra & 28 & 28 & 0 & & & & & \\
\hline Karnal & 41 & 50 & 23 & 0 & & & & \\
\hline Panipat & 62 & 74 & 44 & 22 & 0 & & & \\
\hline Hisar & 128 & 118 & 101 & 97 & 88 & 0 & & \\
\hline Rohatak & 111 & 140 & 110 & 72 & 47 & 56 & 0 & \\
\hline Jind & 92 & 94 & 64 & 52 & 42 & 47 & 36 & 0 \\
\hline \multicolumn{9}{|c|}{ (c) In Nautical Miles } \\
\hline Yamuna Nagar & 0 & & & & & & & \\
\hline Ambala Cantt & 58 & 0 & & & & & & \\
\hline Kurukshetra & 24 & 25 & 0 & & & & & \\
\hline Karnal & 36 & 43 & 20 & 0 & & & & \\
\hline Panipat & 53 & 64 & 38 & 19 & 0 & & & \\
\hline Hisar & 111 & 102 & 87 & 84 & 76 & 0 & & \\
\hline Rohatak & 97 & 122 & 95 & 62 & 41 & 48 & 0 & \\
\hline Jind & 80 & 81 & 56 & 45 & 37 & 41 & 31 & 0 \\
\hline
\end{tabular}

III. ROAD CHARACTERSTICS AND DISCUSSION

A road characteristic is the road attributes which is used by system users to select the route. These characteristics of road are divided into two parts: primary characteristics and secondary characteristics. Influence of the primary characteristics in route selection is more than the secondary characteristics. The presented work uses both characteristics in route selection to achieve reliable results. The primary characteristics include travel distance, travel time, congestion, degree of difficulty, economy, facilities, user friendly and driver stress level. These eight characteristics are widely used in route selection while other are secondary characteristics. They may include- number of turns, traffic signals, road condition, unnecessary detours, traffic incidents, weather, catastrophe, bridge clearance, lane width, bridge load, speed limit etc. Table III shows the importance of road attributes for transportation mode. Most of the attributes are very important for one kind of transport mode while less important for others as explained in table given below.
First two PA like travel time and travel distance are the most important attributes from all other attributes in navigational route selection system. Experience based fuzzy scores are assigned to each road attribute. The fuzzy score 1 is assigned to denote the shortest travel time on a route among the set of feasible routes and a score of 0 is used to denote the longest travel time on a route. Travel distance attribute is similar to the travel time attribute. The fuzzy score 1 of this attribute denotes the shortest travel distance among the set of feasible routes and fuzzy score 0 denotes the longer travel distance. Several causes of road congestion are accidents, road construction, traffic light etc. More congestion on road is denoted by high fuzzy score and low score represents the less congestion on road. Degree of difficulty includes narrowness of road, traffic signals, number of turns, road construction etc. which is difficult for the drive.

A fuzzy score 1 indicates ideal road situation, means easy to drive and score 0 indicates difficult to drive. For example National Highways 1, State Highways 0.8, Urban Road 0.6, 
Rural Road 0.3. Economy includes toll plaza, vehicle maintenance cost, fuel consumption etc. A fuzzy score 1 denotes best economy (no toll, low maintenance cost, low fuel consumption) and 0 denotes the worst economy (maximum toll, high maintenance cost, high fuel consumption). Facilities include the available facilities (filling station, automobile station, restaurant, tea shop, ATM machines etc.) on a road. A fuzzy score 1 denotes that more facilities are available on a route and 0 denotes that there is no facility on route. A score 1 denotes that route is more user friendly and low stress level of the driver and 0 score represents that the route is less user friendly and high stress level of the driver.

TABLE III. IMPORTANCE Wise ClASSIFICATION OF ROAD ATtRIBUtES FOR TRANSPORTATION MODE

\begin{tabular}{|c|c|c|c|c|c|}
\hline & Train & Truck & Bus & Car & Motor Cycle \\
\hline \multicolumn{6}{|l|}{ Primary Attributes (PA) } \\
\hline Travel Distance & & $\mathrm{VM}$ & $\mathrm{VM}$ & $\mathrm{VM}$ & $\mathrm{VM}$ \\
\hline Travel Time & VM & VM & VM & VM & VM \\
\hline Congestion & $V M$ & VM & VM & I & LI \\
\hline Degree of Difficulty & 1 & VM & VM & I & LI \\
\hline Economy & $\mathrm{LI}$ & VM & VM & VM & LI \\
\hline Facilities & $\mathrm{LI}$ & I & VM & $\mathrm{VM}$ & $\mathrm{VM}$ \\
\hline User Friendly & LI & NA & NA & NA & NA \\
\hline Driver Stress Level & $\begin{array}{l}\text { NA } \\
\text { LI }\end{array}$ & VM & $\mathrm{VM}$ & I & I \\
\hline \multicolumn{6}{|c|}{ Secondary Attributes (SA) } \\
\hline Road Condition & NR & VM & VM & VM & \multirow{9}{*}{$\begin{array}{l}\text { LI } \\
\text { LI } \\
\text { LI }\end{array}$} \\
\hline Unnecessary Detours & LI & VM & VM & I & \\
\hline Traffic Incidents & NR & VM & VM & $\mathrm{VM}$ & \\
\hline Weather & VM & VM & VM & VM & \\
\hline Catastrophe & VM & VM & VM & VM & \\
\hline Bridge Clearance & VM & VM & VM & I & \\
\hline Lane Width & NR & VM & VM & I & \\
\hline Bridge Load & VM & VM & VM & I & \\
\hline Speed Limit & VM & VM & VM & VM & \\
\hline
\end{tabular}

Legend: VM- Very Important I - Important

LI- Less Important NR- Not Related NA- Not Analysis

TABLE IV. PRIMARY AND SECONDARY ATtRIBUtes VAluES

\begin{tabular}{|c|c|c|c|c|}
\hline & Route1 & Route2 & Route3 & Route4 \\
\hline $\begin{array}{l}\text { Primary Attributes (PA) } \\
\text { Travel Distance (km) } \\
\text { Travel Time (min) } \\
\text { Congestion } \\
\text { Degree of Difficulty } \\
\text { Economy } \\
\text { Facilities } \\
\text { User Friendly } \\
\text { Driver Stress Level }\end{array}$ & $\begin{array}{l}80 \\
90 \\
\text { No } \\
\text { No } \\
\text { Very Low } \\
\text { More } \\
\text { More } \\
\text { Low }\end{array}$ & $\begin{array}{l}90 \\
110 \\
\text { High } \\
\text { Minor } \\
\text { High } \\
\text { Few } \\
\text { Less } \\
\text { Medium }\end{array}$ & $\begin{array}{l}85 \\
140 \\
\text { Low } \\
\text { Major } \\
\text { Very High } \\
\text { No } \\
\text { Less } \\
\text { High }\end{array}$ & $\begin{array}{l}75 \\
160 \\
\text { No } \\
\text { Minor } \\
\text { Low } \\
\text { No } \\
\text { Medium } \\
\text { Very High }\end{array}$ \\
\hline $\begin{array}{l}\text { Secondary Attributes (SA) } \\
\text { Road Condition } \\
\text { Unnecessary Detours } \\
\text { Traffic Incidents } \\
\text { Bad Weather } \\
\text { Catastrophe } \\
\text { Bridge Clearance (ft.) } \\
\text { Lane Width (ft.) } \\
\text { Bridge Load (pound) } \\
\text { Speed Limit (kmph) }\end{array}$ & $\begin{array}{l}\text { Best } \\
\text { Few } \\
\text { Medium } \\
\text { More Suitable } \\
\text { Low } \\
\geq 15 \\
\geq 14 \\
9000 \\
100\end{array}$ & $\begin{array}{l}\text { Good } \\
\text { Medium } \\
\text { More } \\
\text { Suitable } \\
\text { Medium } \\
\geq 15 \\
\geq 11 \\
5000 \\
80\end{array}$ & $\begin{array}{l}\text { Average } \\
\text { More } \\
\text { More } \\
\text { Not Suitable } \\
\text { High } \\
\geq 10 \\
\geq 9 \\
5000 \\
60\end{array}$ & $\begin{array}{l}\text { Worst } \\
\text { Few } \\
\text { Few } \\
\text { Not Suitable } \\
\text { Medium } \\
\geq 9 \\
\geq 9 \\
3000 \\
50\end{array}$ \\
\hline
\end{tabular}

The acceptable values for some other road attributes (bridge clearance, lane width, bridge load and speed limit) are $\geq 15 \mathrm{ft}$., $\geq 10 \mathrm{ft}$., 9000 pounds and $\geq 40 \mathrm{kmph}$ respectively.

The routes which satisfy these conditions will be assigned high score, otherwise low score will be given to them.

\section{OPTIMAL ROUTE SELECTION CRITERIA}

As discussed in previous section, a driver can select a route based on primary and secondary road attributes. Two types of the query systems (General and Special) are designed here. In general query system the results are returned on the basis of primary road attributes whereas in special query, the results are returned on the basis of both the road attributes. 
The experience based weight is assigned to each road attributes as shown in Table $\mathrm{V}$. These weights are assigned on the basis of road attribute values as given in Table IV. The fuzzy logic is used to select the optimum route for designed system as shown in Fig. 4. In this section a decision making route selection methodology for drivers is presented to select the optimum route for vehicle navigation system using fuzzy logic which is shown in Fig. 4. At first setting of the number of road attributes (primary and secondary) is done then the values of each attributes which are normalized between 0 and 1 are noted, after that classification of road attributes is made for transportation mode. Set the fuzzy rules to each road attribute and assign the experience based fuzzy score to each road attribute.

Rank is assigned to each feasible route on the basis of attribute score. The two input membership function $\mathrm{f}(\mathrm{x})$ and $\mathrm{g}(\mathrm{x})$ are represented in Fig. 5. Here $\mathrm{f}(\mathrm{x})$ is used to show "LOW", "SHORT", "MINIMUM", "FEW" and "MINOR" and g(x) shows "HIGH", "LONG", "MAXIMUM", "MORE", "MAJOR". Five trapezoidal membership functions- "VERY BAD", "BAD", "FAIR", "GOOD" and "VERY GOOD" as shown in Fig. 6. An example of primary and secondary attributes based fuzzy rules is as follows:

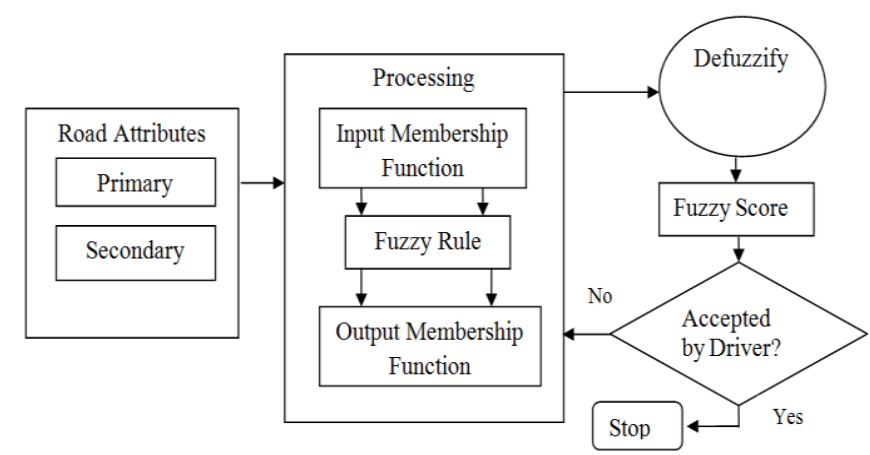

Fig. 4. Optimum route selection methodology

Primary Attribute based Fuzzy Rules as Follow: Rule 1:

IF Travel distance is SHORT on Route1 AND

THEN Choose Route1 AND Route2 should not be taken

TABLE V. FUZZY SCORE OF ROAD ATtRIBUTES

\begin{tabular}{|c|c|c|c|c|}
\hline & Route1 & Route2 & Route3 & Route4 \\
\hline \multicolumn{5}{|l|}{ Primary Attributes (PA) } \\
\hline Travel Distance & & & 0.6 & \\
\hline Travel Time & 0.8 & 0.5 & 0.6 & 0.9 \\
\hline Congestion & 1.0 & 0.9 & 0.8 & 0.4 \\
\hline Degree of Difficulty & 1.0 & 0.1 & 0.1 & 0.5 \\
\hline Economy & 1.0 & 0.9 & 0.4 & 0.7 \\
\hline Facilities & 0.9 & 0.2 & 0.0 & 0.7 \\
\hline User Friendly & 1.0 & 0.4 & 0.2 & 0.0 \\
\hline Driver Stress Level & 1.0 & 0.2 & 0.7 & 0.5 \\
\hline & 1.0 & 0.8 & & 0.2 \\
\hline PA Average Score & & & 0.4 & \\
\hline \multirow{2}{*}{\multicolumn{5}{|c|}{ Secondary Attributes (SA) }} \\
\hline & & & & \\
\hline Road Condition & 1.0 & 0.8 & 0.5 & 0.1 \\
\hline Unnecessary Detours & 0.9 & 0.5 & 0.2 & 0.9 \\
\hline Traffic Incidents & 0.5 & 0.2 & 0.2 & 0.8 \\
\hline Bad Weather & 0.9 & 0.6 & 0.0 & 0.0 \\
\hline Catastrophe & 0.8 & 0.6 & 0.4 & 0.6 \\
\hline Bridge Clearance & 1.0 & 1.0 & 0.7 & 0.5 \\
\hline Lane Width & 1.0 & 0.8 & 0.4 & 0.4 \\
\hline Bridge Load & 1.0 & 0.6 & 0.6 & 0.3 \\
\hline Speed Limit & 0.9 & 0.8 & 0.6 & 0.4 \\
\hline SA Average Score & 0.8 & 0.6 & 0.4 & 0.4 \\
\hline Total Average Score $(\mathrm{PA}+\mathrm{SA})$ & 0.8 & 0.5 & 0.4 & 0.4 \\
\hline
\end{tabular}

Rule 2:

IF

THEN

Rule 3:
IF

Travel time is LARGE on Route1 AND

Travel time is SHORT on Route2

Choose Route2 AND Route1 should not be taken
THEN

Secondary Attribute based Fuzzy Rules as Follow: 
Rule 1:

IF

THEN

Rule 2:

IF

THEN

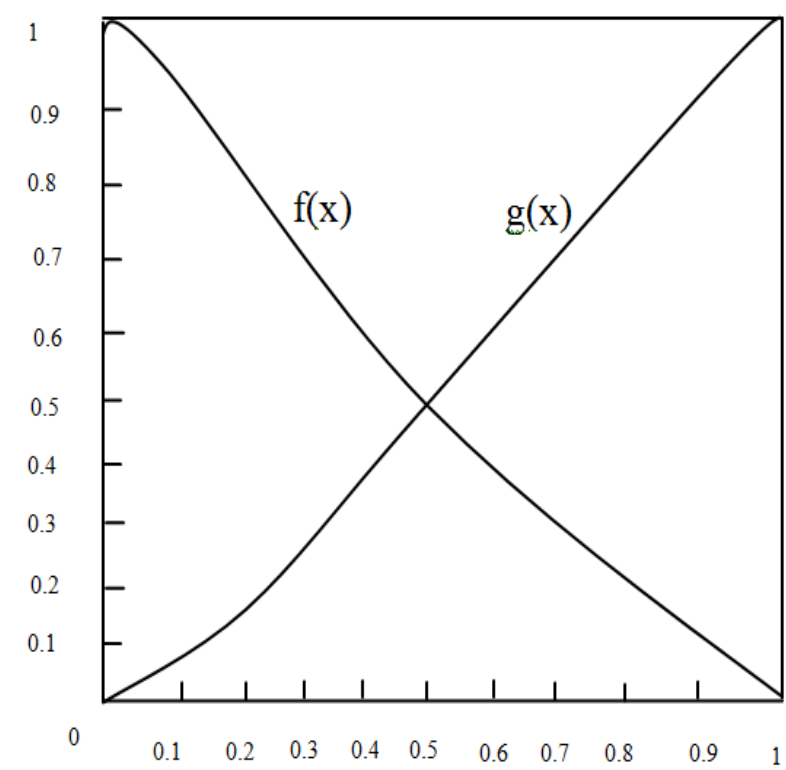

Fig. 5. Input membership functions $\mathrm{f}(\mathrm{x})$ and $\mathrm{g}(\mathrm{x})$ here $\mathrm{f}(\mathrm{x})$ for ("LOW", "SHORT", "MINIMUM", "FEW", "MINOR") and $\mathrm{g}(\mathrm{x})$ for ("HIGH", "LONG", "MAXIMUM", "MORE", "MAJOR")

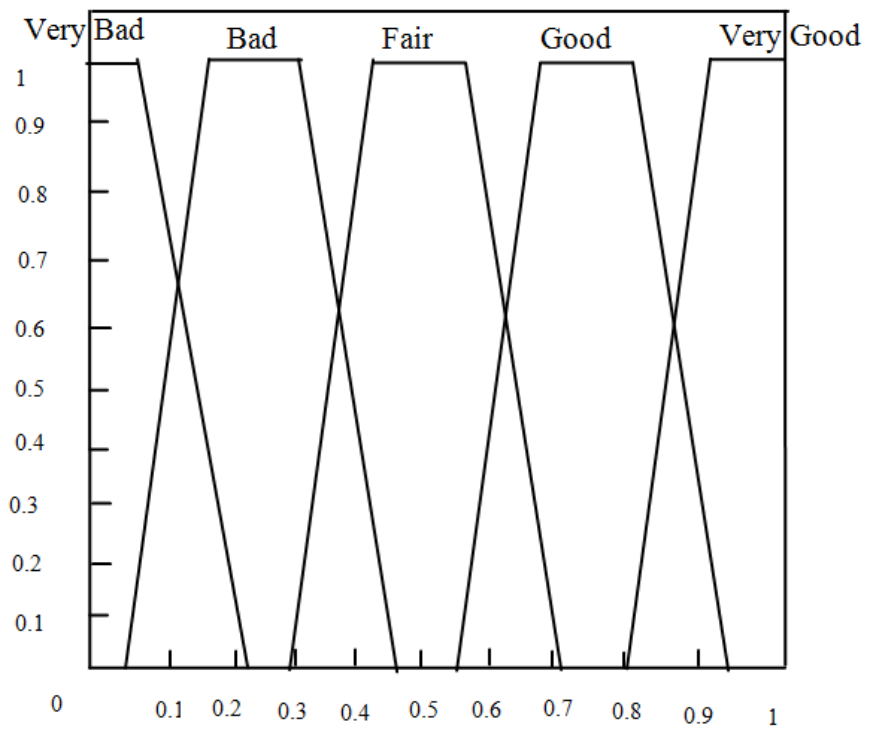

Fig. 6. Output Trapezoidal membership functions for ("VERY BAD",,BAD","FAIR","GOOD","VERY GOOD”)

\section{RESUlT AND DISCUSSION}

A route selection criterion is based on fuzzy concept. The designed system is tested over Haryana route and alternate routes are considered from origin to destination. The program for developed system is written in programming language (PHP, MYSQL) and it is designed for two types of queries (general and special). In case of general query, the designed system returned the results on the basis of average score of PA and in case of special query; the average score of $(\mathrm{PA}+\mathrm{SA})$ is used to display results as shown in Table VI. The system users can view all alternate routes from origin to destination just by entering starting and ending point of journey as well as they can select optimal route for their journey. Four feasible routes and their descriptions are given here in Table I and Table V. Route 1 and Route 2 could be a route with heavy use of National Highway and the average score of primary and secondary attributes is 0.8 on Route1 as shown in Table VI which is the largest score among four feasible routes.

Fuzzy rules are used to specify the optimal route:

Rule 1:

IF

THEN

Rule 2:

IF

THEN

Travel distance is SHORT on Route1 AND Travel distance is LONG on Route2 Route 1 is VERY GOOD and Route2 is very BAD

(

Distance is LONG and Congestion is LOW on Route 1 AND

Distance is SHORT and Congestion is VERY HIGH on Route2

Route 1 is GOOD and Route2 is VERY BAD

TABLE VI. AVERAge SCORE OF PA, SA AND (PA+SA) CORRESPONDING ROUTES

\begin{tabular}{llll}
\hline & PA Score & SA Score & (PA+SA) Score \\
\hline Route1 & 0.9 & 0.8 & 0.8 \\
Route2 & 0.6 & 0.5 & 0.5 \\
Route3 & 0.4 & 0.4 & 0.4 \\
Route4 & 0.4 & 0.4 & 0.4 \\
\hline
\end{tabular}

It's quite clear that Route1 will be optimal route for general and special types of driver's queries. The average score of PA, SA attribute is $0.9,0.8$ respectively of Route 1 and average score of $(\mathrm{PA}+\mathrm{SA})$ is 0.8 which is highly fuzzy score among all alternate routes, so it is recommended route for the driver. Route2 has the next highest score it will be the next recommended route. The designed system provides reliable result as compared to other existing system because almost all road attributes are considered here to assign score to each route.

\section{CONCLUSION AND FUTURE WORK}

An intelligent route selection system is presented in this article. The system provides the optimum route for particular drivers to his/her preference. The concept of primary and secondary road attributes is discussed and a criterion for optimum route selection is set. The route selection criteria is 
based on fuzzy logic in which output Trapezoidal membership functions for Route1, Route2, Route3 and Route4 are " GOOD", "FAIR", "BAD" and "BAD" respectively. The first output membership function shows the optimal route among all alternate routes. This vehicle navigation system will be helpful for drivers in decision making to select more precise optimal route. In future ORSVNS will be a part of more intelligent route selection system for transportation.

\section{REFERENCES}

[1] Grantham K.H. Pang, K. Takahashi,T. Yokota and H. Takenaga," Adaptive Route Selection for Dynamic Route Guidance System Based on Fuzzy-Neural Approaches", IEEE Transactions on Vehicular Technology, Vol. 48, no. 6, November 1999.

[2] A brief report on Auto and Auto Ancillaries in India, ASA \& Associates LLP, July2015.

[3] Dunn Engineering Associates, P.E., Consulting Services," Alternate Route Handbook", U.S Department of Transportation, Federal Highway Administration, May 2006.

[4] Peter Bonsall," The influence of route guidance advice on route choice in urban networks", OKluwer Academic Publishers, Springer, Vol. 19(1), pp. 1-23, 1992.

[5] Mohamed Abdel-Aty and M. Fathy Abdalla," Modeling drivers' diversion from normal routes under ATIS using generalized estimating equations and binomial probit link function", Kluwer Academic Publishers. Printed in the Netherlands, Vol. 31(3), pp. 327-348, 2004.

[6] Alexander Aved, Tai Do, Georgiana Hamza-Lup, Ai Hua Ho, Lap Hoang, Liang Hsia, Kien A. Hua, Fuyu Liu, Rui Peng," A real-time route diversion management system “,Intelligent Transportation Systems Conference, IEEE, pp. 1131-1136, 2007.

[7] M. Shou-Ren HU, Chung-Yung WANG, Chih-Peng CHU and KenChen LEE," Value of traffic information for route diversion control scheme under traffic incidents "Journal of the Eastern Asia Society for Transportation Studies, Vol. 6, pp. 2487 - 2501, 2005.

[8] Kim, Dong Sun," A dynamic route diversion model on urban freeway O-
D pattern predicton", Journal of the eastern Asia Society for Transportation studies, Vol.5, October 2003.

[9] Constantinos Antonioua, Haris N. Koutsopoulos, Moshe Ben-Akiva and Akhilendra S. Chauhan," Evaluation of diversion strategies using dynamic traffic assignment", Transportation Planning and Technology Vol. 34( 3), pp. 199-216, April 2011.

[10] Hey Ran Kim and Kyung Soo Chon," Modeling En-route diversion behavior under on-site traffic information", Journal of the eastern Asia society for transportation studies, Vol. 6, pp. 1833-1843, 2005.

[11] Anastasia Spiliopoulou, Maria Kontorinaki, Ioannis Papamichail and Markos Papageorgiou," Real-Time Route Diversion Control at Congested Motorway off-Ramp Areas - Part I: User Optimum Route Guidance", Proceedings of the 16th International IEEE Annual Conference on Intelligent Transportation Systems (ITSC 2013), The Hague, The Netherlands, October 6-9, 2013.

[12] A. Spiliopoulon, M. Kontorinaki, I. Papamichail and $M$. Papaageorgious," Real-time route diversion control at congested offRamp areas partII: Route guidance versus off-ramp closure", Transportation: can we do more with less resources? transportation. Procedia - social and behavioral sciences. Elsevier Ltd., Vol. 111(5): pp. 1102-1111, 2013.

[13] Xingang Li, Yakang Cao, Xiaomei Zhao, and Dongfan Xie," Drivers' Diversion from Expressway under Real Traffic Condition Information Shown on Variable Message Signs ",KSCE Journal of Civil Engineering, Vol. 9(7), pp. 2262-2270, 2014.

[14] Mohd Rapik Saat and Jesus Aguilar Serrano," Multicriteria high-speed rail route selection: application to Malaysia's high-speed rail corridor prioritization", Transportation Planning and Technology, Taylor \& Francis,Vol. 38(2), pp. 200-213, 2015.

[15] S.S. Keshkamat, J.M. Looijen and M.H.P. Zuidgeest," The formulation and evaluation of transport route planning alternatives: a spatial decision support system for the Via Baltica project, Poland", Journal of Transport Geography, Elsevier Ltd., Vol. 17, pp. 54-64, 2008.

[16] Grantham K.H. Pang, K. Takahashi,T. Yokota and H. Takenaga," Intelligent Route Selection for In-Vehicle Navigation Systems", Transportation Planning and Technol., Vol. 25 (3), pp. 175-213, 2002. 